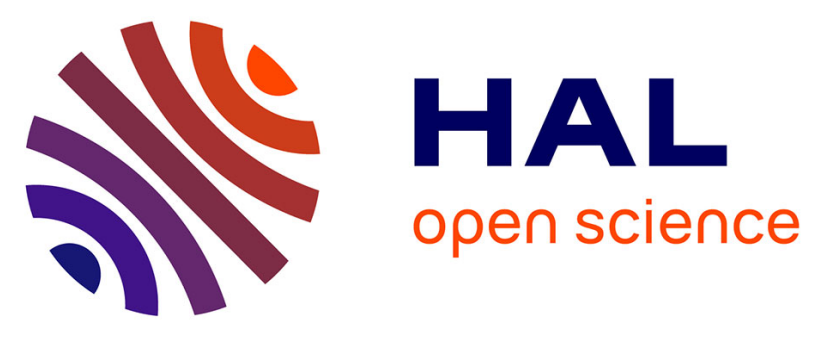

\title{
Temperature dependence of optical properties of InAs/InP quantum rod-nanowires grown on Si substrate
} Mohamed Helmi Hadj Alouane, Olfa Nasr, Hammadi Khmissi, Bouraoui Ilahi, Gilles Patriarche, Mohamad Mahmoud Ahmad, Michel Gendry, C. Bru-Chevallier, Nicolas Chauvin

\section{To cite this version:}

Mohamed Helmi Hadj Alouane, Olfa Nasr, Hammadi Khmissi, Bouraoui Ilahi, Gilles Patriarche, et al.. Temperature dependence of optical properties of InAs/InP quantum rod-nanowires grown on Si substrate. Journal of Luminescence, 2021, 231, pp.117814. 10.1016/j.jlumin.2020.117814 . hal03040324

\section{HAL Id: hal-03040324 \\ https://hal.science/hal-03040324}

Submitted on 4 Dec 2020

HAL is a multi-disciplinary open access archive for the deposit and dissemination of scientific research documents, whether they are published or not. The documents may come from teaching and research institutions in France or abroad, or from public or private research centers.
L'archive ouverte pluridisciplinaire HAL, est destinée au dépôt et à la diffusion de documents scientifiques de niveau recherche, publiés ou non, émanant des établissements d'enseignement et de recherche français ou étrangers, des laboratoires publics ou privés. 


\title{
Temperature dependence of optical properties of InAs/InP quantum rod-nanowires grown on Si substrate
}

\author{
Mohamed Helmi Hadj Alouane a,b,*, Olfa Nasr ${ }^{\mathrm{a}, \mathrm{b}}$, Hammadi Khmissi ${ }^{\mathrm{b}, \mathrm{c}}$, Bouraoui Ilahi ${ }^{\mathrm{b}, \mathrm{d}}$, Gilles Patriarche ${ }^{\mathrm{e}}$, \\ Mohamad Mahmoud Ahmad a, Michel Gendryf,a, Catherine Bru-Chevallier ${ }^{\mathrm{f}, 1}$, Nicolas Chauvin ${ }^{\mathrm{f}, 1}$
}

a Department of Physics, College of Science, King Faisal University, P.O Box: 400, Al-Ahsa, 31982, Saudi Arabia

${ }^{\mathrm{b}}$ Micro-Optoelectronic and Nanostructures Laboratory, Faculty of Sciences, University of Monastir, Monastir, 5019, Tunisia

c Physics Department, Faculty of Science, Northern Border University, Arar, 91431, Saudi Arabia

dinstitut Quantique et Laboratoire Nanotechnologies et Nanosystèmes (LN2), UMI 3463 CNRS, 3IT, Université de Sherbrooke, Sherbrooke, QC, Canada

e Centre de Nanosciences et de Nanotechnologies (C2N)-UMR 9001 CNRS, 10 Boulevard Thomas Gobert, 91120, Palaiseau, France

${ }^{\mathrm{f}}$ Université de Lyon, Institut des Nanotechnologies de Lyon (INL)-UMR, 5270, CNRS, France

\section{A R T I C L E I N F O}

\section{Keywords}

III-V nanowires

Silicon substrates

Monolithic integration

Quantum rods

Telecom-band emitters

FEM

\begin{abstract}
A B S T R A C T
The emergence of semiconductor nanowires (NWs) as a new class of functional materials has generated a great interest in the scientific community in the fields of electronics, photonics and energy. In this work, we report on the optical properties of telecom-band emitting InAs/InP quantum rod-nanowires (QR-NWs) grown on silicon substrates by gold catalyst assisted molecular beam epitaxy (MBE). The energies of A and B band transitions in wurtzite InAs QRs are numerically evaluated by finite element method (FEM) as a function of the QR geometry and strain and compared with the experimental results obtained from photoluminescence (PL). Temperature-dependent optical properties of the QR-NWs are studied revealing that the integrated PL intensity keeps up to $30 \%$ of its value at $14 \mathrm{~K}$ which testify a high stability of the PL intensity. Furthermore, the investigated nanostructure shows a room temperature emission wavelength at $1.55 \mu \mathrm{m}$. These results demonstrate a great promise for telecom-band III-V nanoemitters monolithically grown on silicon.
\end{abstract}

\section{Introduction}

III-V nanowires (NWs), such as InAs, GaAs or InP, are ideal for photonic devices due to their direct band gap and superior electrical properties [1]. These NWs have been proposed for a wide variety of applications ranging from interconnects to functional device elements in electronic and optoelectronic applications [2-6]. Such structures are typically fabricated by a bottom-up approach using the Vapor-Liquid-Solid (VLS) method [7]. The NW growth using VLS method is well adapted to grow III-V semiconductors on silicon (Si) substrate which enables III-V NW optoelectronic devices to be integrated with the established Si microelectronics technology [8]. In contrast to the stable zinc blende (ZB) phase in bulk III-V materials, the Au catalyst growth of III-V NWs occurs preferentially in the hexagonal wurtzite (WZ) phase [9-11] in which the valence band splits in 3 bands, the so called A, $B$ and $C$ bands [12]. Furthermore, NWs with built-in homojunctions or heterojunctions, realized by variation of doping and composition along their length, constitute a new class of promising one dimensional semi- conductor heterostructures $[13,14]$. The insertion of low dimensional quantum heterostructures in NWs adds new degrees of design freedom for optimization of electrical and optical performances [15]. Generally, the VLS approach allows for the growth of quantum rods of any desired height without the limits inherent to the growth of InAs quantum dots using the Stranski-Krastanov (SK) growth mode [16]. Accordingly, InP NWs with InAs QRs are particularly interesting for fiber optic communication applications through the ability to reach the $1.55 \mu \mathrm{m}$ emission wavelength at room temperature [9]. The difference on the crystal lattice between the InAs QR and the InP NW produces an elastic strain which affects the optoelectronic properties of such nanostructures [17]. To ensure efficient optoelectronic devices operating at room temperature, a clear understanding of the effect of temperature on the optical properties of such structure is also highly required. In this context, we report on the optoelectronic properties of InAs/InP quantum rod-nanowires (QR-NWs) grown on Si substrates using the finite element method (FEM) taking into account the strain effect and we investigate their temperature dependent photoluminescence (TDPL) properties.

\footnotetext{
* Corresponding author. Department of Physics, College of Science, King Faisal University, P.O Box: 400, Al-Ahsa, 31982, Saudi Arabia.

E-mail address: malouane@kfu.edu.sa (M.H. Hadj Alouane)

a Ecole Centrale de Lyon, 36 avenue Guy de Collongue, 69,134 Ecully

1 INSA-Lyon, 7 avenue Jean Capelle, 69,621 Villeurbanne, France.
} 


\section{Materials and methods}

The InAs/InP QR-NWs are grown on $\mathrm{Si}(001)$ substrates by solid-source molecular beam epitaxy (ssMBE) and VLS method using Au-In droplets obtained by dewetting of a Au-In film at $700{ }^{\circ} \mathrm{C}[18,19]$. The NWs are grown with a V/III beam equivalent pressure (BEP) ratio of 19. The growth begins with $2 \mathrm{~min}$ of InP at $460{ }^{\circ} \mathrm{C}$. A growth interruption of $5 \mathrm{~s}$ is realized by closing the indium shutter. During this interruption, the phosphorus flux is switched to arsenic flux. Then, the growth of the InAs insertion is done by opening the indium shutter for a growth time of $20 \mathrm{~s}$ at $500{ }^{\circ} \mathrm{C}$. After a $5 \mathrm{~s}$ growth interruption for the arsenic/ phosphorus flux switching, the final InP axial growth is done by opening the indium shutter for a growth duration of $2 \mathrm{~min}$ at $460{ }^{\circ} \mathrm{C}$. The structure is completed with $10 \mathrm{~min}$ of InP radial growth at $340{ }^{\circ} \mathrm{C}$. For the photoluminescence (PL) measurements, the samples are mounted in a closed-cycle temperature-controlled He cryostat, where the temperature can be varied from 14 to $300 \mathrm{~K}$, and excited by a continuous-wave off-resonant excitation. The spectra are collected by a liquid nitrogen-cooled InGaAs detector with a cutoff wavelength at $2.2 \mu \mathrm{m}$ using a conventional lock-in technique.

\section{Results and discussion}

The morphology and structure of the QR-NWs are studied by transmission electron microscopy (TEM) imaging. Fig. 1(a) shows a schematic of the investigated InAs/InP QR-NWs grown on the Si substrate. The NW diameter and length are in the $80-100 \mathrm{~nm}$ and $1.5-2 \mu \mathrm{m}$ range, respectively. The length and diameter of the InAs QRs are in the range of 45-135 nm and 8-12 nm, respectively. These NWs have a WZ structure with the [0001] axis (c-axis) along the NW growth direction for both InP and InAs materials (Fig. 1(e)). Due to the high V/III BEP ratio, no stacking faults or cubic segments were observed in the QR-NWs (Fig. 1(b-e)). Similar structures are studied by SEM and show the typical four $<111>$ directions emerging from the (001) Si $[20,21]$ : four directions are forming a $35^{\circ}$ angle with the Si surface and a $90^{\circ}$ angle from each other, and this result was also observed by our group [22]. In order to improve our understanding of the optical properties of such InAs/InP QR-NWs, low temperature PL spectroscopy experiments under different excitation powers were performed (Fig. 2).

At low excitation power, the PL spectrum shows a single peak around $0.87 \mathrm{eV}$ (peak A) attributed to the ground state emission of the InAs QRs. The full width at half maximum (FWHM) is about $60 \mathrm{meV}$ for this peak. The low temperature inhomogeneous broadening of the spectra is associated with the InAs QR size dispersion, which is consistent with the TEM results. When the excitation power is increased, a second peak (peak B) appears at higher energy $(\sim 0,95 \mathrm{eV})$. According to our TEM studies, the QR diameter D is 4-14 times shorter than the QR length. The direct consequence of this specific geometry is that the quantum confinement of the first electron and hole states (called ground states) are strongly related to the QR diameter. In QRs, the energy spacing between the ground states and the first excited states is small when $\mathrm{L}$ »D. This is confirmed by the work of different groups which have already investigated the electron and hole states in such QRs (which are also called quantum posts or columnar QDs) [23-25]. Moreover, a $70 \mathrm{meV}$ energy spacing has been predicted for a cylindrical InAs/InP QR with a diameter of $9.6 \mathrm{~nm}$ and a height of $18 \mathrm{~nm}$ [26]. As a consequence, the $80 \mathrm{meV}$ energy spacing between the peak A and peak B cannot be related to the energy spacing between the ground and first excited states in our D $10 \mathrm{~nm}$ and $\mathrm{L}>45 \mathrm{~nm}$ QRs. and another explanation has to be found.

In order to get additional information on the origin of this peak, the WZ InAs/InP QR-NWs have been numerically investigated by finite element method (FEM) by solving the single band effective mass Schrodinger equation in cylindrical coordinates taking into account the strain effects $[27,28]$. The nnnth transition energy can be obtained as follow:

$E_{n}=e_{n}+E_{A, B}^{I n A s}+h_{n}$ where $e_{n}$ and $h_{n}$, are the electron and hole confined energies, respectively. $E_{A, B}^{I n A s}$ is the A or B strained InAs band gap energy. The strain dependence of $E_{A}^{I n A s}$ is given by Ref. [29-31]:

$$
\begin{aligned}
E_{A}^{I n A s}=E_{A}^{0 I n A s}+\left(a_{c z}-D_{1}-D_{3}\right) \varepsilon_{z z} & \left(a_{c t}-D_{2}\right. \\
& \left.-D_{4}\right) \varepsilon_{\perp}
\end{aligned}
$$

where $E_{A}^{0 I n A s}$ is the A unstrained band gap energy and $a_{c z}, a_{c t}$ and $D_{i}$ are the deformation potentials of WZ InAs. $\varepsilon_{\perp}=\left(\varepsilon_{r r}+\varepsilon_{\theta \theta}\right)$ and $\varepsilon_{z z}$ are the strain components parallel and perpendicular to the c-axis, respectively. If the QR is approximated by a cylinder, the strain components can be described by the Eshelby model $[17,32,33]$ :

$\left\{\begin{array}{l}\varepsilon_{r r}=\varepsilon_{\theta \theta}=f_{c s}-f_{\text {shear }} \phi\left(\frac{L}{D}\right) \\ \varepsilon_{z z}=f_{c s}+2 f_{\text {shear }}\left[\phi\left(\frac{L}{D}\right)-1\right]\end{array}\right.$

where $f_{c s}$ is the lattice mismatch perpendicular to the NW axis defined by $f_{c s}=\frac{a_{I n P}-a_{I n A s}}{a_{I n A s}}$ were $a_{I n P}$ and $a_{I n A s}$ are the lattice constant of InP and InAs, respectively. The quantity $f_{\text {shear }}$ is defined by: $f_{\text {shear }}=\frac{1}{2} f_{c s} \frac{3 \lambda+2 \mu}{\lambda+2 \mu}$ , where $\lambda$ and $\mu$ are the Lame coefficients. For an isotropic crystal, $\mu=\frac{1}{2}\left(c_{11}-c_{12}\right)$ and $\lambda=c_{12} . \phi\left(\frac{L}{D}\right)$ represents the shape factor where $\mathrm{D}$ is the diameter and $\mathrm{L}$ the length of the $\mathrm{QR}$. $\phi(x)$ is given by the following analytical formula [34]:

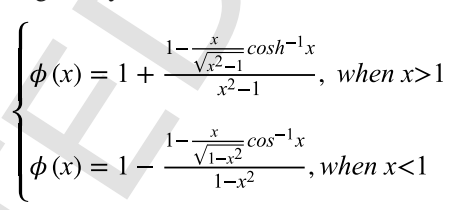

Fig. 3(a) shows the values of $\varepsilon_{\mathrm{rr}}$ and $\varepsilon_{\mathrm{zz}}$ as a function of the QR aspect ratio (L/D). Finally, the energy difference between $A$ and $B$ band energies, $\Delta_{A B}$,is deduced from the following equation [30,31]:

$\Delta_{A B}$

$=\frac{\Delta_{c r}}{2}+\frac{\Delta_{s o}}{2}+\frac{D_{3}}{2} \varepsilon_{z z}+\frac{D_{4}}{2} \varepsilon_{\perp}$
$-\sqrt{\left[\frac{\Delta_{c r}-\frac{1}{3} \Delta_{s o}+D_{3} \varepsilon_{z z}+D_{4} \varepsilon_{\perp}}{2}\right]^{2}+\frac{2}{9} \Delta_{s o}^{2}}$

where $\Delta_{c r}, \Delta_{s o}$ are the crystal field and spin orbit splittings. The value of the deformation potentials, crystal field, spin orbit splitting and unstrained band gap energies used in the calculations are listed in Table 1. All these values are theoretical values with the exception of the WZ InAs band gap being experimentally measured in Refs. [35].

Fig. 3(b) shows the QR aspect ratio dependence of the A and B band gap energies. For infinite QR $\left(\frac{L}{D}>>1\right)$, the energy difference between A and B band gap energies remains constant. In the other hand, for flat $\mathrm{QR}\left(\frac{L}{D}<1\right)$, the $\mathrm{A}$ and $\mathrm{B}$ band gap energies are strongly affected by the strain effect. In our case, the calculations are based on WZ InP NWs with infinite length and diameter. Fig. 4(a) shows the schematic structure of the band alignment of the WZ InAs QR inserted in a WZ InP NW used in our calculations.

The parameters used in the energy level calculations are given in Table 2 , where $m_{e}^{*}, m_{A}^{*}$ and $m_{B}^{*}$ are the effective masses for the electron, A-band hole and B-band hole in $m_{o}$ units (free electron mass), respectively. According to the selection rule in NWs with WZ structure, the emission from recombination of electrons in the conduction band with holes from A-band is expected to be polarized perpendicularly to the $\mathrm{NW}$ axis, while for the recombination with holes from B-band, both perpendicular and horizontal polarizations are allowed $[12,18]$. In that 
(a)
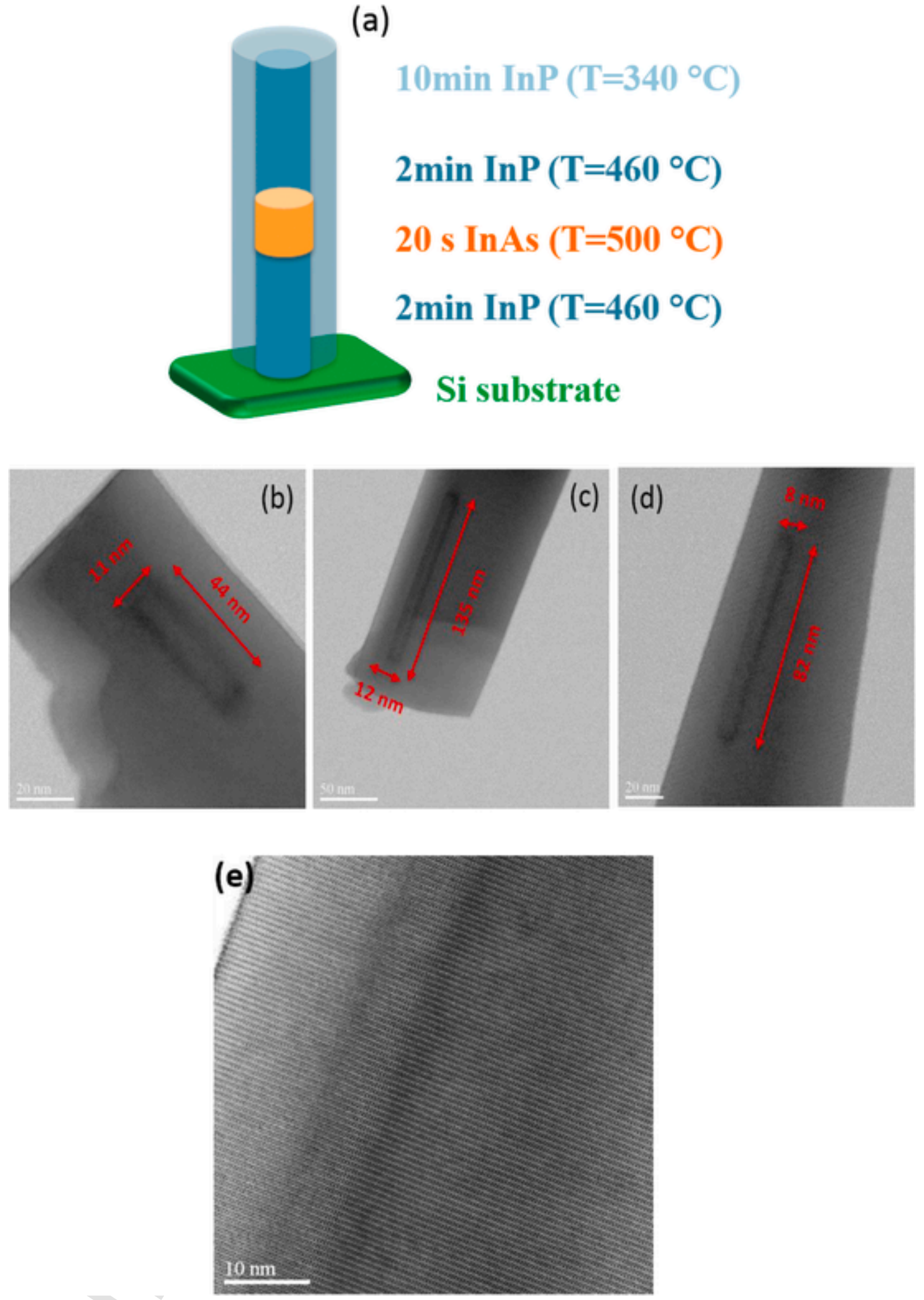

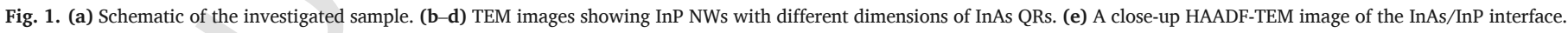

case, the effective masses perpendicular to the NW axis are used in our calculations.

The ground state energies of A and B transitions as a function of the InAs QR diameter and length are represented in Fig. 4(b).
The transition energy is found to exhibit a strong variation up to a QR length around $15 \mathrm{~nm}$. However, no major contribution is observed for longer QRs. When the QR length increases from $2 \mathrm{~nm}$ to $60 \mathrm{~nm}$, the energy spacing between the A and B transition energies decreases from $\approx 114 \mathrm{meV}$ to $\approx 23 \mathrm{meV}$. Whereas a transition from heavy hole-like to 


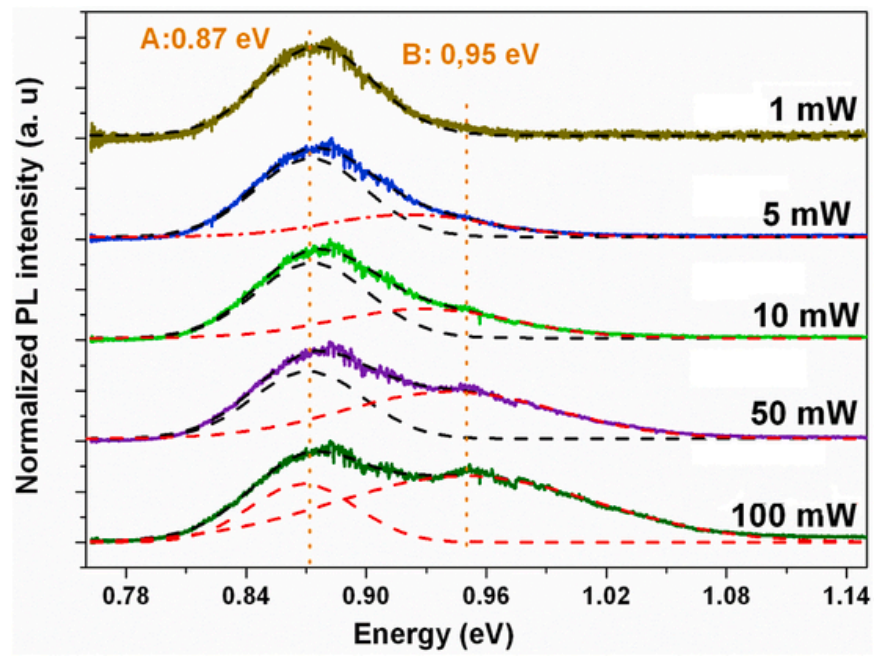

Fig. 2. Normalized PL spectra at $14 \mathrm{~K}$ of InAs/InP QR-NWs as a function of the excitation power. The solid lines are the experimental data and the dotted lines are the Gaussian fit of the PL spectra.

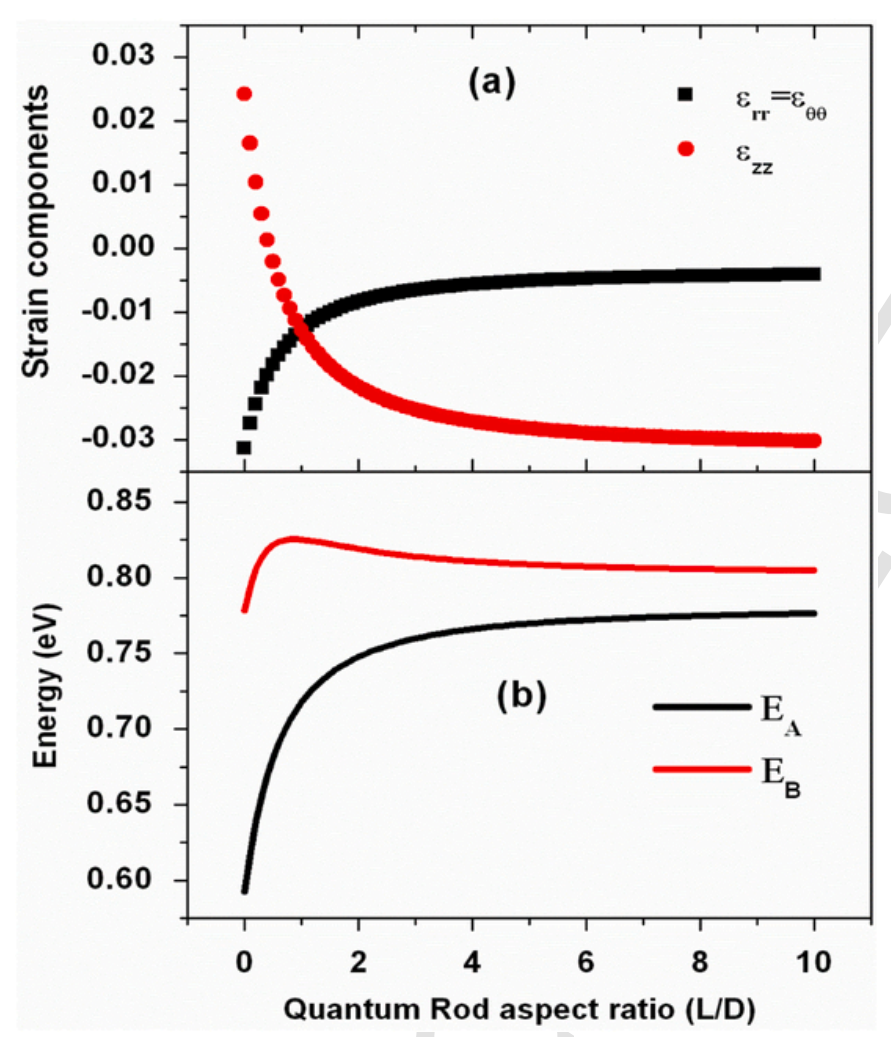

Fig. 3. (a) Strain components and (b) Energies of the strained A and B band gap energies, as a function of the $\mathrm{QR}$ aspect ratio $(\mathrm{L} / \mathrm{D})$.

light hole-like ground state is predicted in ZB InAs QRs [16], in WZ structures the ground state emission is always related to A-band holes. By comparing the calculated and the experimental peak energies, a QR diameter of about $10-14 \mathrm{~nm}$ and an average QR length of more than
$15 \mathrm{~nm}$ can be deduced. In that case, InP NWs with larger QR diameter greater than $10 \mathrm{~nm}$ (Fig. 1(b)-1(c)) dominate the PL spectra. As far as the theoretical results are concerned, several phenomena could explain the discrepancy: the piezoelectric field [19], the strain dependence of the effective masses [37] and the need to take into account the exciton binding energy. Furthermore, a discrepancy is observed in the literature on the theoretical values of some fundamental parameters of WZ InAs $[29,35,36,38]$.

To ensure efficient optoelectronic device operating at room temperature, a clear understanding of the effect of temperature on the optical properties of such structure is highly required. Fig. 5 shows the PL spectra of the sample from $14 \mathrm{~K}$ to $300 \mathrm{~K}$ using low laser excitation power.

The PL emission peak is shifted toward higher wavelength, up to $1.55 \mu \mathrm{m}$ at room temperature with a PL intensity keeping as much as $30 \%$ of its value at $14 \mathrm{~K}$. This figure is close to the best values (50-67\%) reported for ZB InAs QDs or quantum dashes grown in an InP matrix $[39,40]$. As the temperature increases, the QR-NW emission peak due to the thermal escape of carriers from A-band to B-band. A multi-Gaussian fitting is performed on each PL spectrum to take into account the fact that two peaks are observed on the spectrum at room temperature with an average energy spacing of $70 \pm 5 \mathrm{meV}$ which is close to the average energy splitting observed between the A- and B-bands at low temperature.

The temperature dependence of the A exciton integrated PL intensity is shown in Fig. 6(a). A fit of the experimental data has been performed using the following multi-channel Arrhenius equation [41]:

$I=\frac{I_{0}}{1+\sum_{i} c_{i} \exp \left(-\frac{E_{i}}{k_{b} T}\right)}$

where i represents the number of involved channels for carrier thermal escape, $k_{b}$ is the Boltzmann constant, $E_{i}$ is the thermal activation energy, $I_{0}$ is the PL intensity at $0 \mathrm{~K}$ and $\mathrm{c}_{i}$ is a constant. We found that the temperature dependence of the integrated PL intensity can be well fitted by considering two escape channels $(\mathrm{i}=2)$. The best fit gives the values of $\mathrm{E}_{1}$ and $\mathrm{E}_{2}$ around $10( \pm 2 \mathrm{meV})$ and $125( \pm 10 \mathrm{meV}) \mathrm{meV}$, respectively. These values are comparable with the findings of $\mathrm{Wu}$ et al. [42] were 14.3 and $73.4 \mathrm{meV}$ activation energies were obtained for GaAs/GaAsP NW quantum dots and associated with the GaAs surface states, which limits the optical properties of the NWs, and to thermal excitation of photocarriers from the GaAs QDs into the GaAsP barriers, respectively. Alonso-Álvarez et al. [43] also reported 11 and $170 \mathrm{meV}$ activation energies for InAs/GaAsP quantum posts (QPs). The lower activation energy is associated to carrier recombination through defects in the quantum structures or their interfaces while the higher activation energy is compatible with the unipolar escape of carriers from the nanostructures to the matrix [43]. If we assume an isotropic dielectric constant, the WZ InAs exciton binding energy can be estimated using the following equation:

$\mathrm{E}_{\mathrm{b}}=\frac{13.58 \mathrm{eV}}{\varepsilon(0)^{2}}\left[\frac{2}{3}\left(\frac{1}{\mathrm{~m}_{\mathrm{e}}^{\perp}}+\frac{1}{\mathrm{~m}_{\mathrm{A}}^{\perp}}\right)+\frac{1}{3}\left(\frac{1}{\mathrm{~m}_{\mathrm{e}}^{\|}}+\frac{1}{\mathrm{~m}_{\mathrm{A}}^{\|}}\right)\right]^{-1}$

where $\varepsilon(0)$ is the dielectric constant (15.15 for ZB InAs [44]), $\mathrm{m}_{\mathrm{e}}$ and $\mathrm{m}_{\mathrm{A}}$ are the electron and A-band hole effective masses that takes in account the existing anisotropy in the WZ InAs effective masses. Using the parameters given in Table 2 , we thus obtain $\mathrm{E}_{\mathrm{b}}=2 \mathrm{meV}$. This value does not differ from that of the free exciton in bulk ZB InAs

Table 1

The deformation potentials $a_{c z}, a_{c t}$ and $D_{i}$, the crystal field splitting $\Delta_{c r}$, spin orbit splitting $\Delta_{s o}$ and the unstrained band gap energy $E_{A}^{0 I n A s}$ (eV) used in calculations.

\begin{tabular}{|c|c|c|c|c|c|c|c|}
\hline$\Delta_{s o}(e V)$ & $\Delta_{c r}(e V)$ & $\boldsymbol{E}_{\boldsymbol{A}}^{0 \text { InAs }}(\mathrm{eV})$ & $\boldsymbol{D}_{4}(\mathrm{eV})$ & $\boldsymbol{D}_{3}(\mathrm{eV})$ & $a_{c t}-D_{2}(e V)$ & $a_{c z}-D_{1}(e V)$ & \\
\hline $0.379[36]$ & $0.195[36]$ & $0.477[35]$ & $-3.17[29]$ & 5.85 [29] & $-8.33[29]$ & $-2.69[29]$ & InAs (WZ) \\
\hline
\end{tabular}


(a)
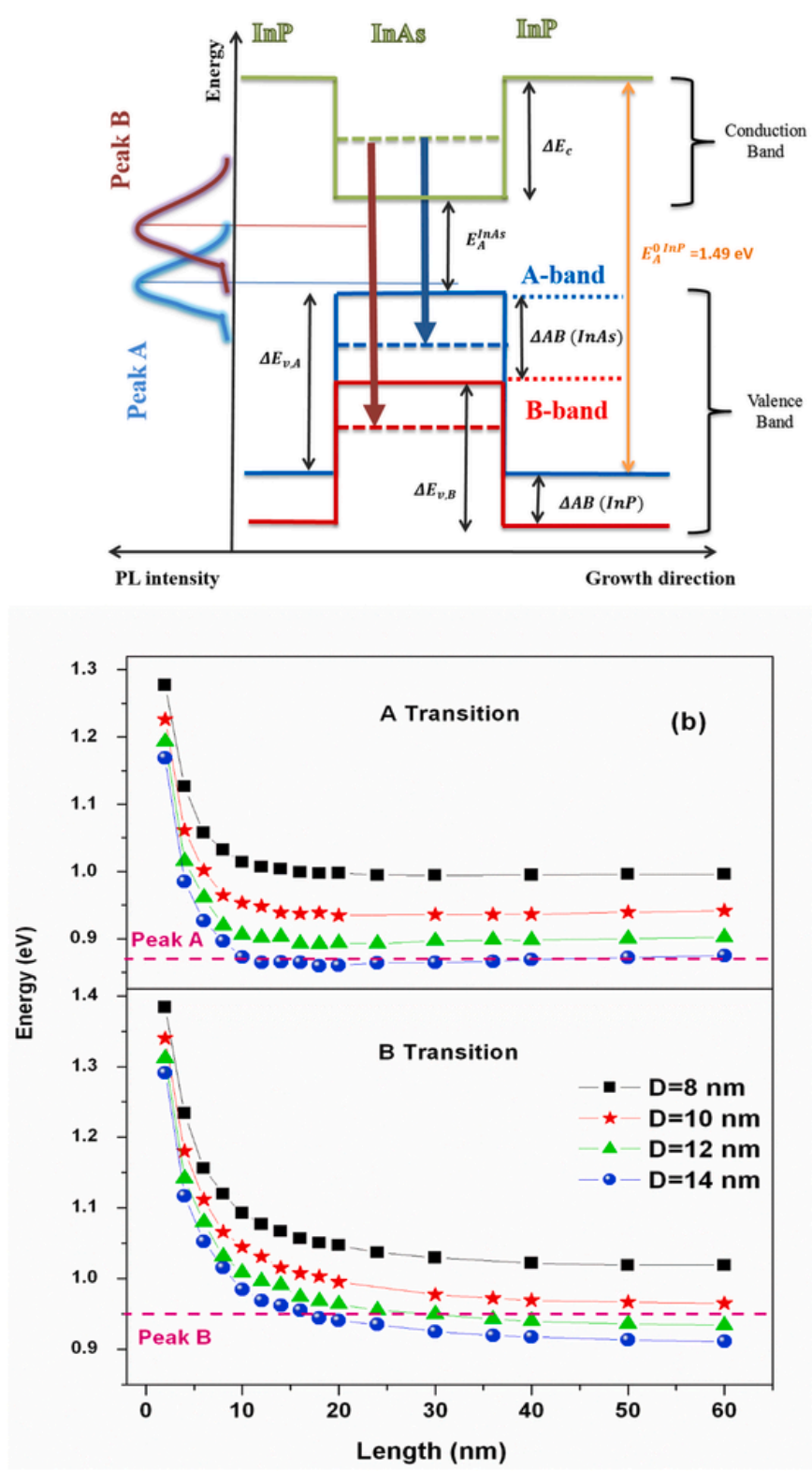

Fig. 4. (a) Schematic representation of the band alignment for WZ InAs/WZ InP used in our calculation. (b) Simulated A and B transition energies of WZ InAs/InP QR-NWs as a function of the QR diameter (D) and length (L) (from $2 \mathrm{~nm}$ to $60 \mathrm{~nm}$ ). Horizontal dashed lines represent the energies of A and B peaks observed by PL.

(1 meV) [44]. Moreover, the exciton binding energy is in the $5-25 \mathrm{meV}$ range for SK InAs/InP QDs $[45,46]$. In that case, the smaller activation energy $E_{1}=10 \mathrm{meV}$ can be associated to the exciton binding energy in WZ InAs. However, the high temperature (160-300 K temperature range) activation energy $\mathrm{E}_{2}$ is about five times less than the difference $(\approx 620 \mathrm{meV}$ ) between the energy of the WZ InP barrier band gap $(1.49 \mathrm{eV})$ and the energy of the PL emission of the A transition
$(0.87 \mathrm{eV})$. In the other hand, this value is close in magnitude to the energy spacing between the A- and B-bands $(80 \mathrm{meV})$. In that case, $\mathrm{E}_{2}$ can be associated to the thermally populated high-energy states in the B-band. This thermal population is confirmed by the relative increase of $\mathrm{B}$ transition PL intensity along with the temperature as observed for WZ InP NWs [47].

Fig. 6(b) shows the FWHM of the A peak as a function of temperature. The FWHM almost remains constant for temperature below $100 \mathrm{~K}$. With further increasing temperature to $160 \mathrm{~K}$, the FWHM was slightly increased by about 5-6 meV and then remains constant at higher temperature range. This behavior has been observed for SK InAs/GaAs QDs and attributed to the thermal population of excited hole states [48]. As far as our sample is concerned, this behaviour might be attributed to the thermal population of the B-band. This is in a good agreement with studies on the temperature dependence of the A transition integrated PL intensity.

The temperature dependence of the emission energy of the A peak is plotted in Fig. 6(c). Generally, this behaviour is discussed in terms of the empirical equation of Varshni [49] and Bose-Einstein formula [50] given by:

$$
\begin{aligned}
& E_{g}(T)=E_{g}(T)+\frac{\alpha T^{2}}{T+\beta} \\
& E(T)=E_{B}-a_{B E}\left[1+\frac{2}{\exp \left(\frac{\theta_{\mathrm{BE}}}{\mathrm{T}}\right)-1}\right]
\end{aligned}
$$

where $\alpha$ and $\beta$ parameters are the Varshni coefficients, aBE represents the strength of the electron-phonon interaction and $\theta_{\mathrm{BE}}$ is the average phonon temperature. Solid and dashed lines in Fig. 6(c) are the fitting curves of the experimental data based on equations (8) and (9), respectively. The best fitting parameters for the two models are summarized in Table 3 along with parameters from the literature. These parameters are closed to the values reported in the literature for ZB InAs which indicates that ZB and WZ InAs have similar thermal parameters as observed for InP [47,54] and GaAs [55] (even if there is some discrepancy for this semiconductor materiel [56]). The low temperature data points $(<100 \mathrm{~K})$ are systematically below the Varshni curves by about $5 \mathrm{meV}$. This value is close in magnitude to the smaller activation energy $E_{1}$ calculated from the fitting of the temperature dependence of the integrated PL intensity by the Arrhenius equation and attributed to the formation of excitons at low temperatures. When the temperature is raised further, the energy shift follows the Varshni law. This constitutes an additional proof of the good quality of our InAs/InP QR-NWs grown on silicon.

\section{Conclusions}

In summary, we have grown high quality InAs/InP QR-NWs on Si(001) substrates by VLS assisted MBE. Optical studies performed at cryogenic temperature reveal an InAs $Q R$ emission in the telecom band with two peaks. FEM calculations taking into account strain effects in the InAs QR were developed to explain these experimental results. Temperature-dependent optical properties revealed a high stability of the PL intensity in the 14-300 K temperature range and showed an emission at the $1.55 \mu \mathrm{m}$ wavelength at room temperature. These results confirm a great promise for InAs-InP QR-NWs for telecom-band na-

Table 2

\begin{tabular}{|c|c|c|c|c|c|c|c|}
\hline & c $(\AA)$ & a $(\AA)$ & $\mathbf{m}_{\mathbf{e}}\left(\mathbf{m}_{0}\right)$ & $\mathbf{m}_{\mathbf{A}}^{\perp}\left(\mathbf{m}_{0}\right)$ & $\mathbf{m}_{\mathbf{A}}^{\|}\left(\mathbf{m}_{0}\right)$ & $\mathbf{m}_{\mathbf{B}}^{\perp}\left(\mathbf{m}_{0}\right)$ & $\mathbf{m}_{\mathbf{B}}^{\|}\left(\mathbf{m}_{0}\right)$ \\
\hline InP (WZ) & 6.77 [29] & 4.15 [29] & $0.088[36]$ & $0.158[36]$ & $1.273[36]$ & $0.169[36]$ & 0.839 [36] \\
\hline InAs (WZ) & 7 [29] & 4.283 [29] & $0.042[36]$ & $0.084[36]$ & $1.7[36]$ & $0.113[28]$ & $0.101[36]$ \\
\hline
\end{tabular}

WZ InAs and WZ InP effective masses and lattice parameters used for the calculations. $\mathrm{m}_{\mathrm{A}, \mathrm{B}}^{\perp}$ and $\mathrm{m}_{\mathrm{A}, B}^{\|}$are the effective masses perpendicular and parallel to the c-axis, respectively. 


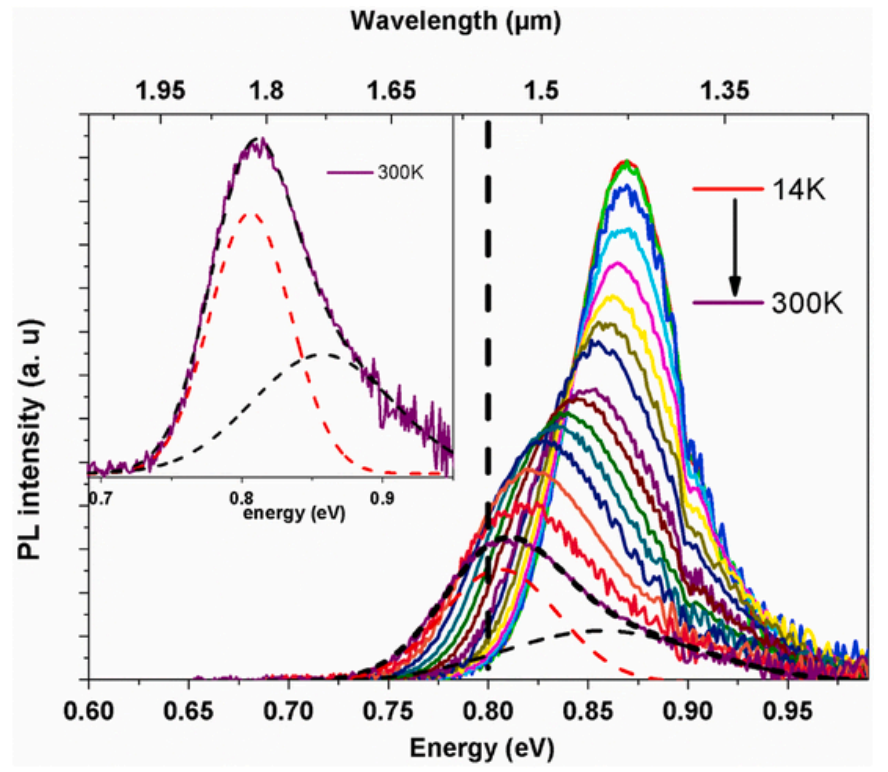

Fig. 5. Temperature induced changes in the PL spectrum for InAs/InP QR-NWs on Si(001) using low laser excitation power. The solid lines are the experimental data and the dotted lines are the multi-Gaussian fitting of the room temperature PL spectra. The vertical dashed line indicates the $1.55 \mu \mathrm{m}$ emission wavelength.

noemitters monolithically integrated on silicon substrates for photonic chips.

\section{Author statement}

Mohamed Helmi Hadj Alouane, Nicolas Chauvin and Catherine Bru-Chevallier carried out the PL measurements on QR-NWs, data analyses, strain calculations and wrote the major part of the paper. Olfa Nasr, Mohamed Hadj Alouane, Mohamad Mahmoud Ahmad and Bouraoui Ilahi contributed in data analyses and FEM calculations. Hammadi Khmissi and Michel Gendry conducted the MBE growth. Gilles Patriarche performed TEM measurements. All authors reviewed the manuscript.

\section{Declaration of competing interest}

The authors declare that they have no known competing financial interests or personal relationships that could have appeared to influence the work reported in this paper.

\section{Acknowledgments}

This work has been supported by the French Agence Nationale pour la Recherche (ANR) "INSCOOP" project. The authors gratefully thank the Nanolyon plateform for access to equipments and J. B. Goure, C. Botella, P. Regreny, P. Cremillieu, and R. Mazurczyk for technical assistance. The authors acknowledge the Deanship of Scientific Research at King Faisal University for financial support under the Research Group Support Track (Grant No. 17122003).

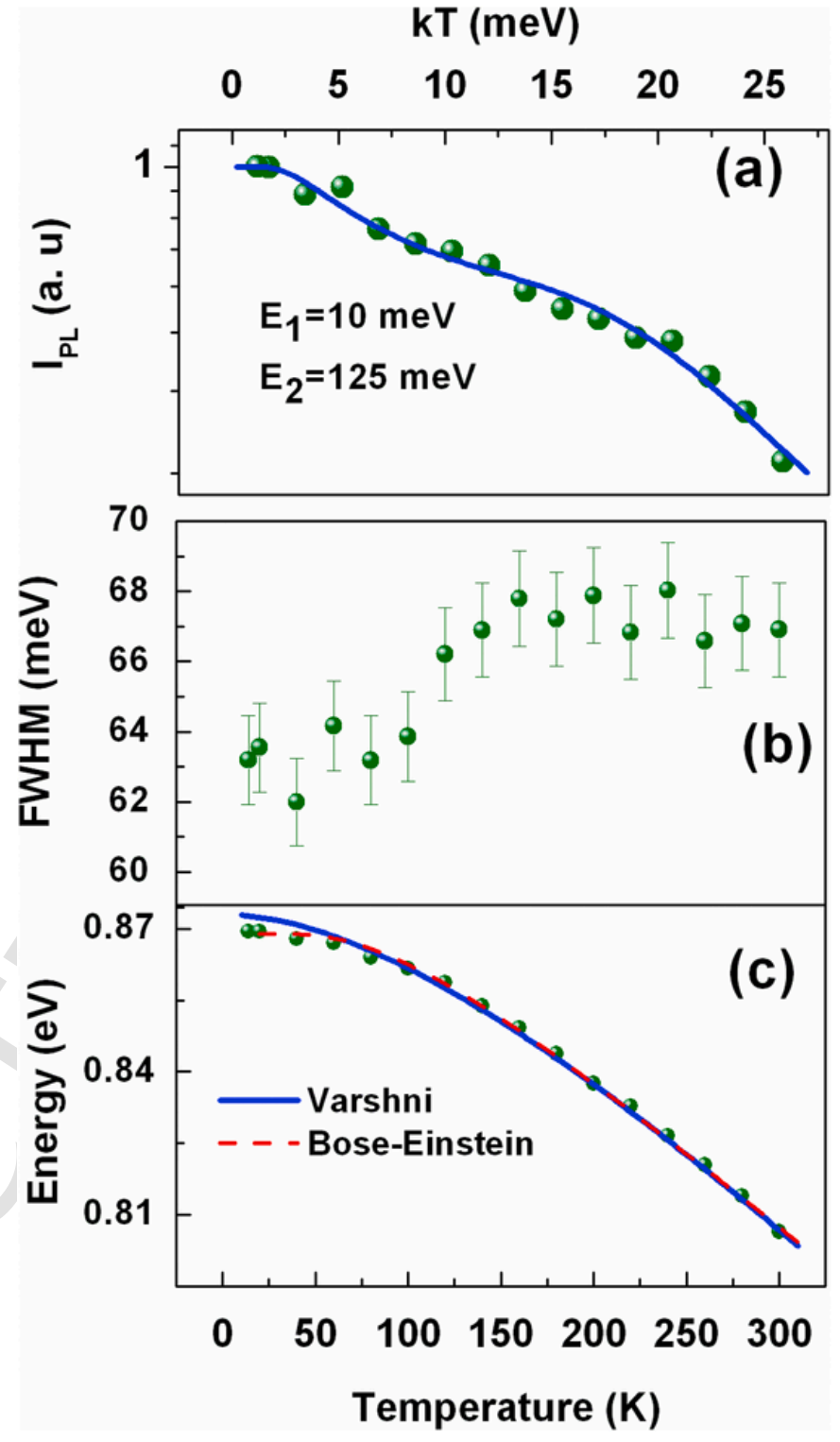

Fig. 6. Temperature dependence of the: (a) integrated PL intensity, (b) FWHM and (c) PL peak energy of A exciton (green circles). The solid curves give the fitting result.

Table 3

Varshni and Bose-Einstein parameters extracted from the fitting of temperature dependence of the band gap energy for InAs/InP QR-NWs.

\begin{tabular}{lllll}
\hline $\boldsymbol{\theta}_{\mathbf{B E}}(\mathrm{K})$ & $\boldsymbol{a}_{\boldsymbol{B E}}(\mathrm{meV})$ & $\beta(\mathrm{K})$ & $\alpha\left(10^{-4} \mathrm{eV} / \mathrm{K}\right)$ & \\
\hline $270 \pm 10$ & $45 \pm 10$ & $270 \pm 10$ & $4.2 \pm 0.5$ & This study \\
$270[52]$ & $99[52]$ & $271[51]$ & $4.19[51]$ & \\
$247[50]$ & $56[50]$ & $93[53]$ & $2.76[53]$ & \\
\hline
\end{tabular}

References

[1] H.J. Joyce, J. Wong-Leung, Q. Gao, H.H. Tan, C. Jagadish, Phase perfection in zinc blende and wurtzite III-V nanowires using basic growth parameters, Nano Lett. 10 (2010) 908-915, doi:10.1021/nl903688v.

[2] H. Sumikura, G. Zhang, M. Takiguchi, N. Takemura, A. Shinya, H. Gotoh, M. Notomi, Mid-infrared lasing of single wurtzite InAs nanowire, Nano Lett. 19 (2019) 8059-8065, doi:10.1021/acs.nanolett.9b03249.

[3] S.J. Gibson, B. Van Kasteren, B. Tekcan, Y. Cui, D. Van Dam, J.E.M. Haverkort, E.P.A.M. Bakkers, M.E. Reimer, Tapered InP nanowire arrays for efficient 
broadband high-speed single-photon detection, Nat. Nanotechnol. 14 (2019) 473-479, doi:10.1038/s41565-019-0393-2.

[4] E. Barrigón, O. Hultin, D. Lindgren, F. Yadegari, M.H. Magnusson, L. Samuelson, L.I.M. Johansson, M.T. Björk, GaAs nanowire pn-junctions produced by low-cost and high-throughput aerotaxy, Nano Lett. 18 (2018) 1088-1092, doi:10.1021/ acs.nanolett.7b04609.

[5] M. Bai, H. Huang, Z. Liu, T. Zhan, S. Xia, X. Li, N. Sibirev, A. Bouravleuv, V.G. Dubrovskii, G. Cirlin, InAs/InP core/shell nanowire gas sensor: effects of InP shell on sensitivity and long-term stability, Appl. Surf. Sci, 498143756 (2019), doi:10.1016/j.apsusc.2019.143756.

[6] D.J. Hill, T.S. Teitsworth, E.T. Ritchie, J.M. Atkin, J.F. Cahoon, Interplay of surface recombination and diode geometry for the performance of axial $\mathrm{p}-\mathrm{i}-\mathrm{n}$ nanowire solar cells, ACS Nano 12 (2018) 10554-10563, doi:10.1021/ acsnano.8b06577.

[7] R.S. Wagner, W.C. Ellis, Vapor-liquid-solid mechanism of single crystal growth, Appl. Phys. Lett. 4 (1964) 89-90, doi:10.1063/1.1753975.

[8] R.L. Woo, R. Xiao, Y. Kobayashi, L. Gao, N. Goel, M.K. Hudait, T.E. Mallouk, R.F. Hicks, Effect of twinning on the photoluminescence and photoelectrochemical properties of indium phosphide nanowires grown on silicon (111), Nano Lett. 8 (2008) 4664-4669, doi:10.1021/nl802433u.

[9] M. Tchernycheva, G.E. Cirlin, G. Patriarche, L. Travers, V. Zwiller, U. Perinetti, J.-C. Harmand, Growth and characterization of InP nanowires with InAsP insertions, Nano Lett. 7 (2007) 1500-1504, doi:10.1021/nl0702281.

[10] M. Mattila, T. Hakkarainen, M. Mulot, H. Lipsanen, Crystal-structure-dependent photoluminescence from InP nanowires, Nanotechnology 17 (2006) 1580-1583, doi:10.1088/0957-4484/17/6/008.

[11] E.D. Minot, F. Kelkensberg, M. van Kouwen, J.A. van Dam, L.P. Kouwenhoven, V. Zwiller, M.T. Borgström, O. Wunnicke, M.A. Verheijen, E.P.A.M. Bakkers, Single quantum dot nanowire LEDs, Nano Lett. 7 (2007) 367-371, doi:10.1021/ nl062483w.

[12] E.G. Gadret, G.O. Dias, L.C.O. Dacal, M.M. De Lima, C.V.R.S. Ruffo, F. Iikawa, M.J.S.P. Brasil, T. Chiaramonte, M.A. Cotta, L.H.G. Tizei, D. Ugarte, A. Cantarero, Valence-band splitting energies in wurtzite InP nanowires: photoluminescence spectroscopy and ab initio calculations, Phys. Rev. B - Condens, Matter Mater. Phys 82 (2010) 125327, doi:10.1103/PhysRevB.82.125327.

[13] M.S. Gudiksen, L.J. Lauhon, J. Wang, D.C. Smith, C.M. Lieber, Growth of nanowire superlattice structures for nanoscale photonics and electronics, Nature 415 (2002) 617-620, doi:10.1038/415617a.

[14] N. Panev, A.I. Persson, N. Sköld, L. Samuelson, Sharp exciton emission from singl InAs quantum dots in GaAs nanowires, Appl. Phys. Lett. 83 (2003) 2238-2240, doi:10.1063/1.1611261

[15] M. Karimi, V. Jain, M. Heurlin, A. Nowzari, L. Hussain, D. Lindgren, J.E. Stehr, I.A. Buyanova, A. Gustafsson, L. Samuelson, M.T. Borgström, H. Pettersson, Room-temperature InP/InAsP quantum discs-in-nanowire infrared photodetectors, Nano Lett. 17 (2017) 3356-3362, doi:10.1021/acs.nanolett.6b05114.

[16] M. Zieliński, Fine structure of light-hole excitons in nanowire quantum dots, Phys. Rev. B - Condens, Matter Mater. Phys 88 (2013) 115424, doi:10.1103/ PhysRevB.88.115424.

[17] D. Ferrand, J. Cibert, Strain in crystalline core-shell nanowires, Eur. Phys, J. Appl. Phys. 67 (2014) 30403, doi:10.1051/epjap/2014140156.

[18] R. Anufriev, N. Chauvin, H. Khmissi, K. Naji, J.-B. Barakat, J. Penuelas, G. Patriarche, M. Gendry, C. Bru-Chevallier, Polarization properties of single and ensembles of InAs/InP quantum rod nanowires emitting in the telecom wavelengths, J. Appl. Phys. 113 (2013), doi:10.1063/1.4804327.

[19] R. Anufriev, N. Chauvin, H. Khmissi, K. Naji, G. Patriarche, M. Gendry, C. Bru-Chevallier, Piezoelectric effect in InAs/InP quantum rod nanowires grown on silicon substrate, Appl. Phys. Lett. 104 (2014) 183101, doi:10.1063/1.4875276.

[20] L. Gao, R.L. Woo, B. Liang, M. Pozuelo, S. Prikhodko, M. Jackson, N. Goel, M.K. Hudait, D.L. Huffaker, M.S. Goorsky, S. Kodambaka, R.F. Hicks, Self-catalyzed epitaxial growth of vertical indium phosphide nanowires on silicon, Nano Lett. 9 (2009) 2223-2228, doi:10.1021/nl803567v.

[21] A.L. Roest, M.A. Verheijen, O. Wunnicke, S. Serafin, H. Wondergem, E.P.A.M Bakkers, Position-controlled epitaxial III-V nanowires on silicon, Nanotechnology 17 (2006) S271, doi:10.1088/0957-4484/17/11/S07.

[22] H. Khmissi, K. Naji, M.H. Hadj Alouane, N. Chauvin, C. Bru-Chevallier, B. Ilahi, G. Patriarche, M. Gendry, InAs/InP nanowires grown by catalyst assisted molecular beam epitaxy on silicon substrates, J. Cryst. Growth 344 (2012) 45-50, doi:10.1016/j.jcrysgro.2012.01.038.

[23] H.J. Krenner, P.M. Petroff, Quantum posts with tailored structural, electronic and optical properties for optoelectronic and quantum electronic device applications, Solid State Commun. 149 (2009) 1386-1394, doi:10.1016/j.ssc.2009.04.037.

[24] R. Nedzinskas, V. Karpus, B. Čechavičius, J. Kavaliauskas, G. Valušis, Electron energy spectrum in cylindrical quantum dots and rods: approximation of separation of variables, Phys. Scripta 90 (2015) 65801, doi:10.1088/0031-8949/ 90/6/065801.

[25] J. Andrzejewski, G. Sȩk, E. O’Reilly, A. Fiore, J. Misiewicz, Eight-band k.p calculations of the composition contrast effect on the linear polarization properties of columnar quantum dots, J. Appl. Phys. 107 (2010) 73509, doi:10.1063/1.3346552.

[26] M. Zieliński, Fine structure of light-hole excitons in nanowire quantum dots, Phys. Rev. B - Condens, Matter Mater. Phys 88 (2013) 115424, doi:10.1103/ PhysRevB.88.115424.

[27] M. Souaf, M. Baira, O. Nasr, M.H.H. Alouane, H. Maaref, L. Sfaxi, B. Ilahi, Investigation of the InAs/GaAs quantum dots' size: dependence on the strain reducing layer's position, Materials 8 (2015) 4699-4709, doi:10.3390/ ma8084699.
[28] R. Nedzinskas, V. Karpus, B. Čechavičius, J. Kavaliauskas, G. Valušis, Electron energy spectrum in cylindrical quantum dots and rods: approximation of separation of variables, Phys. Scripta 90 (2015) 65801, doi:10.1088/0031-8949/ 90/6/065801.

[29] C. Hajlaoui, L. Pedesseau, F. Raouafi, F. Ben Cheikhlarbi, J. Even, J.M. Jancu, First-principles density functional theory study of strained wurtzite InP and InAs, J. Phys. D Appl. Phys. 46 (2013) 505106, doi:10.1088/0022-3727/46/50/ 505106

[30] H.Y. Peng, M.D. McCluskey, Y.M. Gupta, M. Kneissl, N.M. Johnson, Shock-induced band-gap shift in GaN: anisotropy of the deformation potentials, Phys. Rev. B Condens, Matter Mater. Phys 71 (2005) 115207, doi:10.1103/ PhysRevB.71.115207.

[31] S. Chuang, C. Chang, K·p method for strained wurtzite semiconductors, Phys. Rev. B - Condens, Matter Mater. Phys. 54 (1996) 2491-2504, doi:10.1103/ PhysRevB.54.2491.

[32] Eshelby J. D, The determination of the elastic field of an ellipsoidal inclusion, and related problems, in: proc. R. Soc. London A Math. Phys. Eng. Sci., 1957: pp. 376-396.

[33] Eshelby J. D, The elastic field outside an ellipsoidal inclusion, in: proc. R. Soc. London A Math. Phys. Eng. Sci., 1959: pp. 561-569.

[34] A. Artioli, Magnetic polaron in (Cd,Mn)Te quantum dot inserted in $\mathrm{ZnTe}$ nanowire, Université Grenoble Alpes (2016). https://tel.archives-ouvertes.fr/tel01424256.

[35] M.B. Rota, A.S. Ameruddin, H.A. Fonseka, Q. Gao, F. Mura, A. Polimeni, A. Miriametro, H.H. Tan, C. Jagadish, M. Capizzi, Bandgap energy of wurtzite InAs nanowires, Nano Lett. 16 (2016) 5197-5203, doi:10.1021/acs.nanolett.6b02205

[36] A. De, C.E. Pryor, Predicted band structures of III-V semiconductors in the wurtzite phase, Phys. Rev. B - Condens, Matter Mater. Phys 81 (2010) 155210 doi:10.1103/PhysRevB.81.155210.

[37] A. Shikanai, T. Azuhata, T. Sota, S. Chichibu, A. Kuramata, K. Horino, S. Nakamura, Biaxial strain dependence of exciton resonance energies in wurtzite GaN, J. Appl. Phys. 81 (1997) 417-424, doi:10.1063/1.364074.

[38] F. Bechstedt, A. Belabbes, Structure, energetics, and electronic states of III-V compound polytypes, J. Phys. Condens. Matter, 25273201 (2013), doi:10.1088/ 0953-8984/25/27/273201.

[39] A. Lévesque, P. Desjardins, R. Leonelli, R.A. Masut, Temperature dependence of the photoluminescence spectra from InAs(P)/InP multilayers containing thick quantum dots: dot-size-dependent carrier dynamics, Phys. Rev. B - Condens, Matter Mater. Phys 83 (2011) 235304, doi:10.1103/PhysRevB.83.235304.

[40] R. Hostein, A. Michon, G. Beaudoin, N. Gogneau, G. Patriache, J.Y. Marzin, I. Robert-Philip, I. Sagnes, A. Beveratos, Time-resolved characterization of InAsPInP quantum dots emitting in the C -band telecommunication window, Appl. Phys. Lett. 93 (2008) 73106, doi:10.1063/1.2965112.

[41] K. Bando, Y. Masumoto, Narrowing of exciton linewidth of a quantum dot with increasing temperature, Phys. Status Solidi 6 (2009) 57-60, doi:10.1002/ pssc. 200879812

[42] J. Wu, A. Ramsay, A. Sanchez, Y. Zhang, D. Kim, F. Brossard, X. Hu, M. Benamara, M.E. Ware, Y.I. Mazur, G.J. Salamo, M. Aagesen, Z. Wang, H. Liu, Defect-free self-catalyzed GaAs/GaAsP nanowire quantum dots grown on silicon substrate, Nano Lett. 16 (2016) 504-511, doi:10.1021/acs.nanolett.5b04142.

[43] D. Alonso-Álvarez, B. Aĺn, J.M. Ripalda, J.M. Llorens, A.G. Taboada, F. Briones, M.A. Roldán, J. Hernández-Saz, D. Hernández-Maldonado, M. Herrera, S.I. Molina, Strain balanced quantum posts, Appl. Phys. Lett. 98 (2011) 173106, doi:10.1063/1.3583455

[44] P. Tang, M. Pullin, C. Phillips, Binding energy of the free exciton in indium arsenide, Phys. Rev. B - Condens, Matter Mater. Phys. 55 (1997) 4376-4381, doi:10.1103/PhysRevB.55.4376.

[45] H. Pettersson, C. Pryor, L. Landin, M. Pistol, N. Carlsson, W. Seifert, L. Samuelson, Electrical and optical properties of self-assembled InAs quantum dots in InP studied by space-charge spectroscopy and photoluminescence, Phys. Rev. B Condens, Matter Mater. Phys. 61 (2000) 4795-4800, doi:10.1103/ PhysRevB.61.4795.

[46] C. Cornet, C. Levallois, P. Caroff, H. Folliot, C. Labb́, J. Even, A. Le Corre, S. Loualiche, M. Hayne, V.V. Moshchalkov, Impact of the capping layers on lateral confinement in InAsInP quantum dots for $1.55 \mu \mathrm{m}$ laser applications studied by magnetophotoluminescence, Appl. Phys. Lett. 87 (2005) 233111, doi:10.1063/ 1.2132527

[47] M.H. Hadj Alouane, N. Chauvin, H. Khmissi, K. Naji, B. Ilahi, H. Maaref, G. Patriarche, M. Gendry, C. Bru-Chevallier, Excitonic properties of wurtzite InP nanowires grown on silicon substrate, Nanotechnology 24 (2013) 35704, doi:10.1088/0957-4484/24/3/035704.

[48] R. Heitz, I. Mukhametzhanov, A. Madhukar, A. Hoffmann, D. Bimberg Temperature dependent optical properties of self-organized InAs/GaAs quantum dots, J. Electron. Mater. 28 (1999) 520-527, doi:10.1007/s11664-999-0105-Z.

[49] Y.P. Varshni, Temperature dependence of the energy gap in semiconductors, Physica 34 (1967) 149-154, doi:10.1016/0031-8914(67)90062-6.

[50] S.D. Singh, S. Porwal, T.K. Sharma, K.C. Rustagi, Temperature dependence of the lowest excitonic transition for an InAs ultrathin quantum well, Cit, J. Appl. Phys. 99 (2006) 63517, doi:10.1063/1.2184431.

[51] S. Paul, J.B. Roy, P.K. Basu, Empirical expressions for the alloy composition and temperature dependence of the band gap and intrinsic carrier density in GaxIn 1-xAs, J. Appl. Phys. 69 (1991) 827-829, doi:10.1063/1.348919.

[52] H. Chouaib, N. Chauvin, C. Bru-Chevallier, C. Monat, P. Regreny, M. Gendry, Photoreflectance spectroscopy of self-organized InAs/InP(0 01 1) quantum sticks emitting at $1.55 \mu \mathrm{m}$, Appl. Surf. Sci. 253 (2006) 90-94, doi:10.1016/ j.apsusc.2006.05.081. 
[53] I. Vurgaftman, J.R. Meyer, L.R. Ram-Mohan, Band parameters for III-V compound semiconductors and their alloys, J. Appl. Phys. 89 (2001) 5815-5875, doi:10.1063/1.1368156.

[54] A. Zilli, M. De Luca, D. Tedeschi, H.A. Fonseka, A. Miriametro, H.H. Tan, C. Jagadish, M. Capizzi, A. Polimeni, Temperature dependence of interband transitions in wurtzite InP nanowires, ACS Nano 9 (2015) 4277-4287, doi:10.1021/acsnano.5b00699.
[55] N. Vainorius, S. Kubitza, S. Lehmann, L. Samuelson, K.A. Dick, M.E. Pistol, Temperature dependent electronic band structure of wurtzite GaAs nanowires, Nanoscale 10 (2018) 1481-1486, doi:10.1039/c7nr07635e.

[56] L. Ahtapodov, J. Todorovic, P. Olk, T. Mjåland, P. Slåttnes, D.L. Dheeraj, A.T.J. van Helvoort, B.-O. Fimland, H. Weman, A story told by a single nanowire: optical properties of wurtzite GaAs,, Nano Lett. 12 (2012) 6090-6095, doi:10.1021/ nl3025714. 